\title{
A Hard Way to the Nucleus
}

\author{
MICHAEL BUKRINSKY
}

As a member of the Retrovirus family, human immunodeficiency virus (HIV), a causative agent of AIDS, replicates by integrating its genome into the host cell's nuclear DNA. However, in contrast to most retroviruses that depend on mitotic dissoIution of the nuclear envelope to gain access to the host cell's genome, the HIV pre-integration complex can enter the nucleus of the target cell during the interphase. Such capacity greatly enhances HIV replication and allows the virus to productively infect terminally differentiated nonproliferating cells, such as macrophages. Infection of macrophages is a critical factor in the pathogenesis of diseases caused by HIV-1 and other lentiviruses. The mechanisms responsible for this unusual feature of HIV have enticed researchers since the early 90s, when the first characterization of the HIV-1 pre-integration complex was reported. Several viral factors, including matrix protein, integrase, viral protein R, and central DNA flap, have been proposed as regulators of HIV-1 nuclear import, only to be later shown as nonessential for this process. As a result, after more than a decade of intense research, there is still no consensus on which HIV-1 and cellular proteins control this critical step in HIV-1 replication. In this review, we will discuss recent advances and suggest possible solutions to the controversial issue of HIV-1 nuclear import.

\section{INTRODUCTION}

Intensive research into the fundamental processes of human immunodeficiency virus type 1 (HIV-1) replication has yielded knowledge that in many respects equals or exceeds that of other retroviruses. As a clearer picture of the pattern of HIV-1 replication evolves, it becomes apparent that HIV-1 biology is distinct from that of the prototypic simple retroviruses in several key aspects, particularly with regard to host cell range and determinants of viral permissiveness. These characteristics are determined to a large extent by the ability of HIV-1 to replicate in nondividing cells $(1,2)$. Until the early 90s, the dogma that retroviruses replicate only in proliferating cells was automatically extended to HIV. The first report convincingly demonstrating that HIV-1 can productively infect nondividing cells came from the Cullen laboratory (3). This work used differentiated macrophages as natural nonproliferating target cells for HIV-1, and macrophages became the object of many subsequent studies dealing with HIV-1 nuclear import. The ability of HIV-1 to establish productive infection in nondividing cells was later confirmed on many other cell types, including T-cell lines and HeLa cells engineered to express HIV-1 receptors, artificially arrested in various phases of the cell cycle $(1,4-7)$. Such cells provided convenient experimental models to investigate molecular mechanisms governing the enigmatic process of HIV-1 nuclear translocation.

The replication cycle of retroviruses involves reverse transcription of genomic RNA in the cytoplasm of an infected cell and integration into the host cell's DNA. To get access to the genomic DNA of a nondividing cell, HIV needs to transport its genome from the plasma membrane to the nucleus and then through an intact nuclear envelope. Inability to cross the nuclear membrane of an interphase cell appears to be the main barrier that restricts replication of simple retroviruses, such as Murine Leukemia Virus (MLV), to proliferating cells (8), where nuclear and cytoplasmic components mix during mitosis. To deliver its genome through the cytoplasm and into the nucleus, HIV-1 most likely uses components of the cellular transport machinery. In recent years, considerable progress has been achieved in characterizing the mechanisms involved in HIV-1 nuclear import. Nevertheless, despite many reports describing possible molecular mechanisms that may regulate delivery of the HIV genome into the nucleus through an intact nuclear membrane, this process remains one of the most controversial topics in HIV biology.

\section{THE JOURNEY OF THE HIV-1 REVERSE TRANSCRIPTION COMPLEX THROUGH THE CYTOPLASM}

Mature, infectious HIV-1 particles contain a characteristic coneshaped core that encases the viral RNA and replication proteins (9). The core shell is made of capsid protein CA p24. After fusing with the target cell, the viral core is released into the cytosol. Whereas reverse transcription can initiate within the core, its disassembly appears to be essential for the progress of reverse transcription. Disassembly of the core is characterized by dissociation of the CA protein $(10,11)$, which is an important step in viral uncoating. Interestingly, the speed of HIV-1 uncoating appears to be much more rapid than that of MLV, where capsid protein stays associated with the pre-integration complexes through reverse transcription and may even be present in the integration-competent

The George Washington University, Washington, D.C. 
pre-integration complex (12). This difference in uncoating between HIV and MLV may be relevant to the pathways these viruses use to access the nucleus, as uncoating is likely to be necessary for the ability of HIV to migrate through the nuclear pore. Uncoating results in formation of a reverse transcription complex (RTC), comprised of HIV-1 RNA and associated viral proteins critical for reverse transcription (reverse transcriptase [RT]), nuclear targeting (matrix protein [MA], viral protein R [Vpr], and integrase [IN]), and integration.

To reach the pores in the nuclear envelope, the RTC must travel from the plasma membrane through the cytoplasm to the nucleus. Because of the high viscosity of the cytoplasm, movement of these particles by diffusion is likely to be very limited, especially considering the size of the HIV-1 RTC, which has been estimated to be at least $50 \mathrm{~nm}$ in diameter (13). To overcome this obstacle, HIV-1 utilizes the cellular cytoskeleton. Initial movements of the virus within the peripheral regions of the cell cytoplasm occur in association with actin cytoskeleton (14). However, subsequent translocation of the HIV-1 pre-integration complex toward the nucleus takes place along the microtubule network (15). Transfer from the actin to the microtubule network is consistent with the evidence that actin can be used to gain access to tubulin (16). The structural basis for interaction between the viral RTC and microtubules remains to be determined. This interaction likely engages a cellular dynein-dependent motor complex, which has both minus end-directed (toward the microtubule-organizing center located close to the nucleus) and plus end-directed (toward cell periphery) motor activities (15). Thus, the virus needs to stimulate the minus end and/or inhibit the plus-end activity to ensure translocation toward the nucleus. A similar mechanism has been demonstrated for the intracellular transport of other viruses, such as herpes simplex virus (HSV)-1 (17) and adenovirus (18).

Reverse transcription occurs during the HIV-1 RTC transport through the cytoplasm and is mostly completed by the time the complex reaches the nucleus. Conversion of the viral genomic RNA into DNA likely leads also to dissociation of reverse transcriptase (Bukrinsky and others, unpublished observation), thus further reducing the size of the complex in preparation for translocation through the nuclear pore. Upon completion of reverse transcription, the complex becomes integration-competent (13) and is termed pre-integration complex (PIC).

\section{PIC ENTRY INTO THE NUCLEUS}

Once near the nuclear membrane, HIV-1 most likely relies on the cellular nuclear import proteins to pass through the nuclear pore. An alternative mechanism based on the ability of Vpr to induce reversible ruptures of the nuclear membrane, which might serve as entry points for the PIC, has been suggested (19); however, this possibility has not been tested experimentally in the context of HIV infection. The model favored by investigators working in the area of HIV nuclear import is that the HIV PIC itself is karyophilic. This implies that a component or components of the complex contain targeting signals that engage the cellular transport proteins, which direct the PIC through the nuclear pore. The most likely candidates for the role of karyophilic agents are viral proteins associated with the PIC (see below), although they may regulate trafficking indirectly by binding host cell-derived karyophilic proteins (20).

Another factor proposed to regulate HIV nuclear import is the specific structure of the viral cDNA intermediate (21). During the plus-strand synthesis of the HIV (and other lentiviruses) cDNA, a 99 nucleotide long "central DNA flap" is produced because of additional initiation of the plus DNA synthesis at another polypurine tract in the middle of the HIV genome. This short trimeric structure was proposed as a critical determinant of the PIC passage through the nuclear pore (21) and was reported to dramatically increase the efficiency of lentivirus-mediated gene transfer $(22,23)$. However, later reports demonstrated that the central flap, at least in some cells, is not essential for PIC nuclear import and its effect is dependent on the HIV-1 strain used $(24,25)$. Most likely, the DNA flap assists the nuclear import function of HIV proteins by providing the optimal conformation to the PIC that is necessary for its interaction with cellular factors and translocation through the nuclear pore.

Three HIV-1 proteins, MA, IN, and Vpr, have been proposed as karyophilic agents that recruit the cellular nuclear import machinery to the PIC. Historically, MA was the first protein implicated in HIV-1 nuclear import $(1,26)$. While most of matrix in the virion localizes outside the core and forms the layer between the viral capsid and the envelope (hence its name), some MA molecules are found in tight association with the HIV core and the PIC $(10,13)$. The HIV-1 MA carries 2 functional, yet rather weak, nuclear localization signals (NLSs) (27), which encode a short stretch of basic amino acids that introduce a positive charge that is crucial for the nuclear targeting properties of these sequences (reviewed in 28). Mutations in MA NLSs significantly attenuate HIV-1 replication in nondividing cells $(1,26,29)$. Import of cellular proteins containing NLSs across the nuclear pore complex is mediated by special transport proteins called importins or karyopherins that bind NLS-containing proteins in the cytosol and target them to the nucleus (30). In the case of basic-type NLSs, such as those present in MA, a heterodimer of 2 importins, importin $\alpha$ and importin $\beta$, is involved. Importin $\alpha$ is actually an adaptor that ensures binding of a basic-NLS-substrate to importin $\beta$, which is then responsible for docking the importin-NLS protein complexes to nucleoporins (a collective term for nuclear pore complex proteins) and translocation through the pore (31). By analogy, it has been postulated that nuclear import of the HIV-1 PIC is also dependent on importins $\alpha$ and $\beta(1,32)$.

However, several later reports questioned the role of MA in HIV-1 nuclear import $(33,34)$. The main argument against MA being the principal HIV nuclear targeting protein was the finding that the virus lacking most of MA was still capable of infecting nondividing cells, albeit with a greatly reduced efficiency (35). While MA is clearly required for efficient nuclear import of the HIV-1 PIC, it appears to be nonessential and is likely only one of several factors regulating this process (35).

A role for IN in HIV-1 nuclear import was originally proposed by Gallay and coworkers (36), who demonstrated that IN interacts with importin $\alpha$ and can target a fusion GST-IN protein into the nucleus of microinjected cells. A more recent report from Malim's group (37) extended this observation and identified an 
unusual NLS spanning residues 161-173 within the central core domain of IN. Mutations within this domain, such as V165A or R166A, disrupted the nuclear import of the integrase. However, later reports were unable to confirm that the 161-173 region of IN functions as an NLS and instead demonstrated that the NLS mutations studied previously primarily affected integration rather than the PIC nuclear import $(24,38)$. Nevertheless, it remains possible that IN is involved in HIV-1 nuclear import, as it is a highly karyophilic protein that can interact with different importins, including importin $\alpha / \beta$ (36) and importin 7 (39). The latter importin (or a heteroduplex of importin 7 and importin $\beta$ ) was found to mediate HIV-1 nuclear import in digitonin-permeabilized macrophages, whereas importin $\alpha$ did not seem to be involved (39). Because importin 7 normally mediates nuclear import of ribosomal protein rpL23a by directly binding to its nonbasic NLS (40), this result also argues against the role of basic NLSs in HIV-1 nuclear import, at least in the digitonin-permeabilized system. Along these lines, it should be noted that while digitoninpermeabilized cells provide a model to study transport of simple cargos through the nuclear envelope, this system is unlikely to recreate the complex pathway of HIV migration into the nucleus of different cell types. Consistent with this view, the level of reduction of HIV-1 infection by silencing of importin 7 using RNAi approach was different in direct comparisons between glioma cells and HeLa cells (39).

Vpr is another HIV-derived protein that has been implicated in nuclear import. Its role in this process was revealed when it was found that the HIV-1 molecular clone, MFD, used in the early studies to investigate HIV-1 nuclear import, encodes a truncated version of the Vpr protein $(41,42)$. Vpr truncation is highlighted by the ability of the HIV-1 IIIB strain, from which the HIV-1 MFD clone was derived, to establish persistent productive infection of T-cell lines (43). Viruses with functional Vpr are unable to do this because of Vpr-induced cell cycle arrest and apoptosis of infected cells (44). Subsequent studies demonstrated that Vpr significantly stimulates HIV-1 infection of nondividing cells, in particular macrophages, presumably by enhancing nuclear import of the PIC $(45,46)$.

Two hypotheses (not necessarily mutually exclusive) for the mode of action of Vpr in HIV-1 nuclear import have been proposed: (1) Vpr targets the HIV-1 PIC to the nucleus via a distinct, importin-independent pathway $(32,47)$; (2) Vpr modifies cellular importin-dependent import machinery $(48,49)$. The 1st model was based on the observation that in the in vitro nuclear import assay Vpr can enter nuclei in the absence of soluble import factors (47). Later reports that Vpr can directly bind nucleoporins (50-52) suggested that Vpr might mediate binding of the PIC to the nuclear pore, thus performing activity normally attributed to impotins. The 2nd model was based on the finding that Vpr can bind to importin $\alpha(49,50)$ and change its affinity for the nuclear localization signal (49). This activity would explain the stimulatory effect of Vpr on nuclear import driven by basic type NLSs through importin $\alpha / \beta$ pathway (49). It would be important to test whether a similar stimulatory activity of Vpr can be detected on nuclear import carried out by other importins.

Most of the experimental data on which these 2 models are based have been obtained using Vpr-expressing vectors. To under-

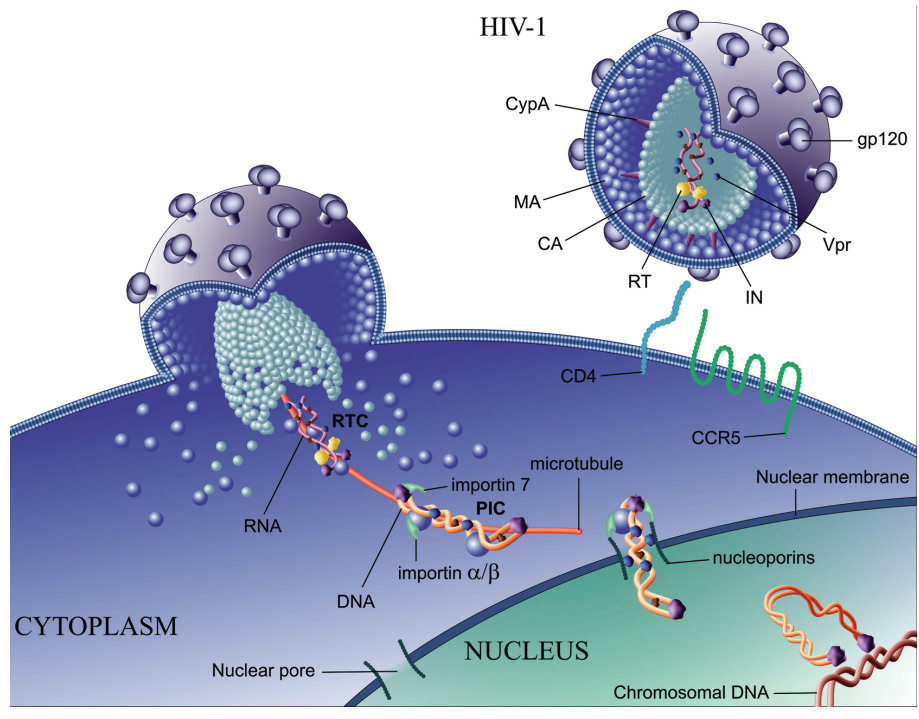

Figure 1. Pre-integration steps of HIV-1 replication. Events starting with HIV-1 binding to its main receptors on the target cell, CD4 and CCR5, and ending with viral integration into the host cell's genome are shown. Proteins packaged into virions are shown; some of these proteins (MA, RT, IN, and Vpr) find their way into the reverse transcription complex (RTC). RTC becomes the pre-integration complex upon completion of reverse transcription. Details of the viral journey through the cytoplasm and into the nucleus are discussed in the text.

stand the actual mechanism of Vpr activity in the nuclear importation of the HIV-1 PIC, studies employing experimental systems dealing with the whole virus or at least the PIC will be required. In any case, Vpr appears to be involved in the process of HIV-1 nuclear import in some cells, in particular macrophages. However, it is obviously not the only player; and its role is not strictly essential, as viruses lacking Vpr can still replicate in macrophages, albeit with reduced efficiency (41).

\section{CONCLUSIONS}

Nuclear import of the HIV-1 PIC appears to be an extremely complex and redundant process. Although each individual factor described in this review might be nonessential, together they ensure the efficiency of this extremely important for the virus step. This probably comes as no surprise, as the ability to replicate in nondividing cells, especially those of the monocyte/macrophage lineage, is critical for survival of non-primate lentiviruses and could be among the main factors driving HIV evolution as well (53). More research is clearly necessary to identify additional cellular proteins involved in HIV-1 nuclear import and understand interaction of these proteins with viral factors. However, certain conclusions can be drawn from currently available data. Based on studies with HIV and other viruses replicating in the nucleus, it seems reasonable to postulate that the HIV-1 PIC is delivered to the nuclear envelope along the microtubule network and enters the nucleoplasm through the nuclear pore. The simplified model depicting the main steps in the process of nuclear translocation of the HIV genome is shown in Figure 1. Upon entry into the cytoplasm, the viral core undergoes initial uncoating characterized by 
dissociation of the capsid protein and formation of the RTC. Further studies are needed to determine molecular characteristics of the RTC, such as the molar ratio of the RTC proteins, their conformation and relative localization in the complex. Such information is critical for understanding interactions between the HIV-1 RTC and cellular proteins.

The RTC associates with tubulin (matrix protein might regulate this step but exact molecular details are still unknown) and is transported toward the nucleus along the microtubule. During this translocation, the viral genomic RNA is converted to a double-stranded DNA and the complex becomes the PIC, which interacts with import receptors (importins) and cellular proteins that may protect viral DNA from degradation and facilitate integration (54). For interaction with importins, viral proteins matrix, integrase, and Vpr are instrumental, but their relative contribution is still uncertain. Vpr may also contribute to the binding of the PIC to nucleoporins. The central DNA flap in the HIV-1 cDNA may provide the optimal structure to the PIC for interaction with importins and/or passage through the nuclear pore. Several different importin molecules, including importin $\alpha / \beta$ and importin 7 , may bind simultaneously to the same PIC and carry it through the nuclear pore (55). The detailed mechanisms responsible for the described steps in the HIV-1 journey through the cell await their resolution. A factor complicating the story is that the molecular details of the HIV nuclear import, such as the type of the importin molecules or the identity of the main viral protein governing interaction with importins, might differ depending on the cell type and infection conditions. This ability of the virus to adapt to various circumstances is likely the reason we almost never get a simple answer to our questions about HIV biology.

Address correspondence and reprint requests to Michael Bukrinsky, George Washington University Medical Center, 2300 Eye Street NW, Ross Hall Room 734, Washington, DC 20037. Phone: 202-994-2036; fax: 202-994-2913; e-mail: mtmmib@gwumc.edu.

Submitted July 24, 2003; accepted for publication October 7, 2003.

\section{REFERENCES}

1. Bukrinsky MI, Haggerty S, Dempsey MP, Sharova N, Adzhubel A, Spitz L, Lewis P, Goldfarb D, Emerman M, and Stevenson M. (1993) A nuclear localization signal within HIV-1 matrix protein that governs infection of non-dividing cells. Nature 365:666-9.

2. Lewis PF, Emerman M. (1994) Passage through mitosis is required for oncoretroviruses but not for the human immunodeficiency virus. J. Virol. 68:510-6.

3. Weinberg JB, Matthews TJ, Cullen BR, Malim MH. (1991) Productive human immunodeficiency virus type 1 (HIV-1) infection of nonproliferating human monocytes. J. Exp. Med. 174:1477-82.

4. Bukrinsky MI, Sharova N, Dempsey MP, Stanwick TL, Bukrinskaya AG, Haggerty S, Stevenson M. (1992) Active nuclear import of human immunodeficiency virus type 1 preintegration complexes. Proc. Natl. Acad. Sci. U.S.A. 89:6580-4.

5. Lewis $P$, Hensel M, Emerman M. (1992) Human immunodeficiency virus infection of cells arrested in the cell cycle. EMBO J. 11:3053-8.

6. Li G, Simm M, Potash MJ, Volsky DJ. (1993) Human immunodeficiency virus type 1 DNA synthesis, integration, and efficient viral replication in growth-arrested T cells. J. Virol. 67:3969-77.

7. Gallay P, Swingler S, Aiken C, Trono D. (1995) HIV-1 infection of nondividing cells: $\mathrm{C}$-terminal tyrosine phosphorylation of the viral matrix protein is a key regulator. Cell 80:379-88

8. Roe T, Reynolds TC, Yu G, Brown PO. (1993) Integration of murine leukemia virus DNA depends on mitosis. EMBO J. 12:2099-108.

9. Briggs JA, Wilk T, Welker R, Krausslich HG, Fuller SD. 2003. Structural organization of authentic, mature HIV-1 virions and cores. EMBO J. 22:1707-15.

10. Bukrinsky MI, Sharova N, McDonald TL, Pushkarskaya T, Tarpley WG, Stevenson
M. (1993) Association of integrase, matrix, and reverse transcriptase antigens of human immunodeficiency virus type 1 with viral nucleic acids following acute infection. Proc. Natl. Acad. Sci. U.S.A. 90:6125-9.

11. Fassati A, Goff SP. (2001) Characterization of intracellular reverse transcription complexes of human immunodeficiency virus type 1. J. Virol. 75:3626-35.

12. Bowerman B, Brown PO, Bishop JM, Varmus HE. (1989) A nucleoprotein complex mediates the integration of retroviral DNA. Genes Dev. 3:469-78.

13. Miller MD, Farnet CM, Bushman FD. 1997. Human immunodeficiency virus type 1 preintegration complexes: studies of organization and composition. J. Virol. 71:5382-90.

14. Bukrinskaya A, Brichacek B, Mann A, Stevenson M. (1998) Establishment of a functional human immunodeficiency virus type 1 (HIV-1) reverse transcription complex involves the cytoskeleton. J. Exp. Med. 188:2113-25.

15. McDonald D, Vodicka MA, Lucero G, Svitkina TM, Borisy GG, Emerman M, Hope TJ. (2002) Visualization of the intracellular behavior of HIV in living cells. J. Cell Biol. 159:441-52.

16. Taunton J. (2001) Actin filament nucleation by endosomes, lysosomes and secretory vesicles. Curr. Opin. Cell Biol. 13:85-91.

17. Sodeik B, Ebersold MW, Helenius A. (1997) Microtubule-mediated transport of incoming herpes simplex virus 1 capsids to the nucleus. J. Cell Biol. 136:1007-21.

18. Suomalainen M, Nakano MY, Keller S, Boucke K, Stidwill RP, Greber UF. (1999) Microtubule-dependent plus- and minus end-directed motilities are competing processes for nuclear targeting of adenovirus. J. Cell Biol. 144:657-72.

19. de Noronha CM, Sherman MP, Lin HW, Cavrois MV, Moir RD, Goldman RD Greene WC. (2001) Dynamic disruptions in nuclear envelope architecture and integrity induced by HIV-1 Vpr. Science 294:1 105-8.

20. Gupta K, Ott D, Hope TJ, Siliciano RF, Boeke JD. (2000) A human nuclear shuttling protein that interacts with human immunodeficiency virus type 1 matrix is packaged into virions. J. Virol. 74:11811-24.

21. Zennou V, Petit C, Guetard D, Nerhbass U, Montagnier L, Charneau P. (2000) HIV-1 genome nuclear import is mediated by a central DNA flap. Cell 101 173-85.

22. Sirven A, Pflumio F, Zennou V, Titeux M, Vainchenker W, Coulombel L, DubartKupperschmitt A, Charneau P. (2000) The human immunodeficiency virus type-1 central DNA flap is a crucial determinant for lentiviral vector nuclear import and gene transduction of human hematopoietic stem cells. Blood 96:4103-10.

23. Van Maele B, De Rijck J, De Clercq E, Debyser Z. (2003) Impact of the centra polypurine tract on the kinetics of human immunodeficiency virus type $1 \mathrm{vec}$ tor transduction. J. Virol. 77:4685-94.

24. Dvorin JD, Bell P, Maul GG, Yamashita M, Emerman M, Malim MH. (2002) Reassessment of the roles of integrase and the central DNA flap in human immunodeficiency virus type 1 nuclear import. J. Virol. 76:12087-96.

25. Limon A, Nakajima N, Lu R, Ghory HZ, Engelman A. (2002) Wild-type levels of nuclear localization and human immunodeficiency virus type 1 replication in the absence of the central DNA flap. J. Virol. 76:12078-86.

26. von Schwedler U, Kornbluth RS, Trono D. (1994) The nuclear localization signal of the matrix protein of human immunodeficiency virus type 1 allows the establishment of infection in macrophages and quiescent T lymphocytes. Proc. Natl. Acad. Sci. U.S.A. 91:6992-6.

27. Nadler SG, Tritschler D, Haffar OK, Blake J, Bruce AG, Cleaveland JS, (1997) Differential expression and sequence-specific interaction of karyopherin alpha with nuclear localization sequences. J. Biol. Chem. 272:4310-5.

28. Dingwall C, Laskey RA. 1991. Nuclear targeting sequences-a consensus? Trends Biochem. Sci. 16:478-81.

29. Haffar OK, Popov S, Dubrovsky L, Agostini I, Tang H, Pushkarsky T, Nadler SG Bukrinsky M. (2000) Two nuclear localization signals in the HIV-1 matrix protein regulate nuclear import of the HIV-1 pre-integration complex. J. Mol. Biol. 299:359-68.

30. Mattaj IW, Englmeier L. (1998) Nucleocytoplasmic transport: the soluble phase. Annu. Rev. Biochem. 67:265-306.

31. Rexach M, Blobel G. (1995) Protein import into nuclei: association and dissociation reactions involving transport substrate, transport factors, and nucleoporins. Cell 83:683-92.

32. Gallay P, Stitt V, Mundy C, Oettinger M, Trono D. (1996) Role of the karyopherin pathway in human immunodeficiency virus type 1 nuclear import. J. Virol. 70:1027-32.

33. Fouchier RA, Meyer BE, Simon JH, Fischer U, Malim MH. (1997) HIV-1 infection of non-dividing cells: evidence that the amino-terminal basic region of the viral matrix protein is important for Gag processing but not for post-entry nuclear import. EMBO J. 16:4531-9.

34. Freed EO, Englund G, Martin MA. (1995) Role of the basic domain of human immunodeficiency virus type 1 matrix in macrophage infection. J. Virol. 69:3949-54

35. Reil H, Bukovsky AA, Gelderblom HR, Gottlinger HG. 1998. Efficient HIV-1 replication can occur in the absence of the viral matrix protein. EMBO J. 17:2699-708.

36. Gallay P, Hope T, Chin D, Trono D. (1997) HIV-1 infection of nondividing cells through the recognition of integrase by the importin/karyopherin pathway. Proc. Natl. Acad. Sci. U.S.A. 94:9825-30.

37. Bouyac-Bertoia M, Dvorin JD, Fouchier RA, Jenkins Y, Meyer BE, WU LI, Emerman M, Malim MH. 2001. HIV-1 infection requires a functional integrase nls. Mol. Cell 7:1025-35.

38. Limon A, Devroe E, Lu R, Ghory HZ, Silver PA, Engelman A. 2002. Nuclear localization of human immunodeficiency virus type 1 preintegration complexes (PICs): $\mathrm{V} 165 \mathrm{~A}$ and R166A are pleiotropic integrase mutants primarily defective for integration, not PIC nuclear import. J. Virol. 76:10598-607. 
39. Fassati A, Gorlich D, Harrison I, Zaytseva L, Mingot JM. (2003) Nuclear import of HIV-1 intracellular reverse transcription complexes is mediated by importin 7 . EMBO J. 22:3675-85

40. Jakel S, Gorlich D. 1998. Importin beta, transportin, RanBP5, and RanBP7 mediate nuclear import of ribosomal proteins in mammalian cells. EMBO J. 17:4491-502.

41. Heinzinger NK et al. (1994) The Vpr protein of human immunodeficiency virus type 1 influences nuclear localization of viral nucleic acids in nondividing host cells. Proc. Natl. Acad. Sci. U.S.A. 91:7311-5.

42. Emerman M, Bukrinsky M, Stevenson M. (1994) HIV-1 infection of non-dividing cells. Nature 369:107-8

43. Dahl K, Martin K, Miller G. (1987) Differences among human immunodeficiency virus strains in their capacities to induce cytolysis or persistent infection of a lymphoblastoid cell line immortalized by Epstein-Barr virus. J. Virol. 61:1602-8.

44. Stewart SA, Poon B, Jowett JB, Chen IS. (1997) Human immunodeficiency virus type 1 Vpr induces apoptosis following cell cycle arrest. J. Virol. 71:5579-92.

45. Connor RI, Chen BK, Choe S, Landau NR. (1995) Vpr is required for efficient replication of human immunodeficiency virus type-1 in mononuclear phagocytes. Virology 206:935-44.

46. Nie Z, Bergeron D, Subbramanian RA, Yao XJ, Checroune F, Rougeau N, Cohen EA. (1998) The putative alpha helix 2 of human immunodeficiency virus type 1 Vpr contains a determinant which is responsible for the nuclear translocation of proviral DNA in growth-arrested cells. J. Virol. 72:4104-15.
47. Jenkins Y, McEntee M, Weis K, Greene WC. (1998) Characterization of HIV-1 Vpr nuclear import: analysis of signals and pathways. J. Cell Biol. 143:875-85.

48. Popov S, Rexach M, Ratner L, Blobel G, Bukrinsky M. (1998) Viral protein R regulates docking of the HIV-1 preintegration complex to the nuclear pore complex. J. Biol. Chem. 273:13347-52.

49. Popov S, Rexach M, Zybarth G, Reiling N, Lee MA, Ratner L, Lane CM, Moore MS, Blobel G, Bukrinsky M. (1998) Viral protein R regulates nuclear import of the HIV-1 pre-integration complex. EMBO J. 17:909-17.

50. Vodicka MA, Koepp DM, Silver PA, Emerman M. (1998) HIV-1 Vpr interacts with the nuclear transport pathway to promote macrophage infection. Genes Dev. 12:175-85.

51. Fouchier RA et al. (1998) Interaction of the human immunodeficiency virus type 1 Vpr protein with the nuclear pore complex. J. Virol. 72:6004-13.

52. Le Rouzic E et al. (2002) Docking of HIV-1 Vpr to the nuclear envelope is mediated by the interaction with the nucleoporin hCG1. J. Biol. Chem. 277:45091-8.

53. Weiss RA. (2003) HIV and AIDS: looking ahead. Nat. Med. 9:887-91.

54. Turelli P et al. (2001) Cytoplasmic recruitment of INII and PML on incoming HIV preintegration complexes: interference with early steps of viral replication. Mol. Cell 7:1245-54.

55. Nermut MV, Fassati A. (2003) Structural analyses of purified human immunodeficiency virus type 1 intracellular reverse transcription complexes. J. Virol. 77:8196-206. 